\title{
Traduire
}

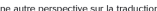

Revue française de la traduction

$241 \mid 2019$

La formation à I'honneur

\section{Formation universitaire et choix traductifs du traducteur juridique}

Bérangère Denizeau

\section{(2) OpenEdition}

1 Journals

Édition électronique

URL : http://journals.openedition.org/traduire/1832

DOI : 10.4000/traduire.1832

ISSN : 2272-9992

Éditeur

Société française des traducteurs

Édition imprimée

Date de publication : 16 décembre 2019

Pagination : 31-43

ISSN : 0395-773X

\section{Référence électronique}

Bérangère Denizeau, «Formation universitaire et choix traductifs du traducteur juridique », Traduire [En ligne], 241 | 2019, mis en ligne le 16 décembre 2019, consulté le 05 février 2020. URL : http:// journals.openedition.org/traduire/1832 ; DOI : 10.4000/traduire.1832 


\section{Formation universitaire et choix traductifs du traducteur juridique}

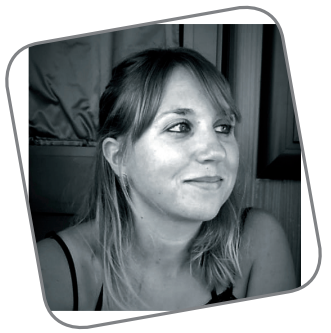

\section{Bérangère Denizeau}

\section{Le «juritraducteur» diplômé en droit et le traducteur juridique diplômé en langues}

Cet article présente les résultats d'une enquête menée auprès de deux groupes de traducteurs travaillant dans la même combinaison linguistique, de l'italien vers le français, et dans le même domaine, le droit. C'est leur formation universitaire qui diffère:

- La moitié d'entre eux sont juristes de formation, bilingues ou ayant une excellente connaissance de l'italien. Nous les appelons ici les «juritraducteurs». Le terme "juritraducteur» provient du néologisme «juritraductologie» qui, selon Jacques Pelage, désigne «une science auxiliaire du droit» au sein de laquelle «[...] l'interprétation du discours est un point de convergence entre le travail du juriste et celui du traducteur $\gg(2001,87)$. 
À la lumière de cette définition, la juritraductologie apparaît d'emblée comme indissociable du droit comparé que Jacques Pelage qualifie d'«acquisition de connaissances thématiques qui enrichiront la culture du traducteur» (Ibid., p. 91).

- L'autre moitié est constituée de linguistes, diplômés d'un master de traduction spécialisée (LEA, etc.) ou ayant acquis des compétences juridiques de manière autodidacte. Nous les appelons les «traducteurs juridiques». L'étude de ces deux profils a donné lieu à une analyse comparée des solutions proposées à la traduction d'un même jugement portant sur la condamnation d'un homme politique italien, Marcello Dell'Utri, arrêté pour ses engagements criminels avec Cosa nostra, la mafia sicilienne.

\section{Difficultés rencontrées par les répondants}

De notre enquête, il ressort que nos traducteurs, indépendamment de leur formation universitaire, se sont retrouvés confrontés à quatre problèmes principaux:

- En premier lieu, ils ont évoqué des difficultés d'ordre terminologique qui contredisent la vision de la traduction juridique comme un acte "mécanique» (Schleiermacher, 1999, 39), consistant à faire coïncider les termes d'une langue A avec ceux d'une langue B. Pour Florence Terral, agrégée de droit, la complexité de la terminologie juridique ne peut être limitée à son aspect technique, car elle se caractérise nécessairement par une empreinte culturelle qui rend impossible un recours systématique à des correspondances terminologiques préétablies $(2004,876)$.

\begin{tabular}{l|l} 
Expression italienne: & $\begin{array}{l}\text { "Voglia il Tribunale, contrariis rejectis, cosi } \\
\text { giudicare:» }\end{array}$ \\
\hline Traduction littérale: & «Veuille le Tribunal, contrariis rejectis, juger ainsi:»
\end{tabular}




\begin{tabular}{|c|c|}
\hline Traduction proposée: & $\begin{array}{l}\text { Solution 1: "Qu'il plaise à la Cour de rejeter les } \\
\text { arguments' et de statuer comme suit:» } \\
\overline{1 . D u} \text { demandeur, de l'intimé et de l'autre partie [NdT]. } \\
\text { Solution } 2 \text { : «Plaise au tribunal désigné, après avoir } \\
\text { débouté la partie adverse de toute conclusion } \\
\text { contraire, de décider ce qui suit:» }\end{array}$ \\
\hline $\begin{array}{l}\text { Commentaires des } \\
\text { traducteurs: }\end{array}$ & $\begin{array}{l}\text { 1: "J'ai dû utiliser une note de bas de page, car } \\
\text { la formule latine, dans le texte italien, permet de } \\
\text { comprendre qu'il s'agit des arguments de la partie } \\
\text { adverse.» } \\
\text { 2: "J'ai utilisé une paraphrase, car la formule latine } \\
\text { est une expression figée en italien.» }\end{array}$ \\
\hline
\end{tabular}

Pour traduire la formule latine, deux solutions ont été proposées: la note de bas de page et la périphrase. La première constitue selon Jean-Louis Cordonnier (1995) la seule manière de rendre un texte de départ accessible à son public sans le modifier: elle invite implicitement le destinataire de la traduction à entreprendre des recherches pour comprendre la portée exacte du terme ayant posé un problème. Néanmoins, elle présente le double risque de modifier la forme du texte d'arrivée, et de transformer le traducteur en «pédagogue polyglotte chargé d'expliquer le droit» (Hadi, $2002,76)$.

- Ensuite, des difficultés d'ordre syntaxique et phraséologique: Gérard Cornu parle de linguistique juridique comme du fait de partir du "choix des mots»pour arriver aux «choix des formulations» $(2005,21)$. Les répondants ont précisément évoqué le problème soulevé par cette phraséologie, définie par Lovis Beaudoin comme l'uensemble des combinaisons préférentielles d'une unité lexicale avec des substantifs, des verbes, des adjectifs» (Beaudoin 2008, 1). 


\begin{tabular}{|c|c|}
\hline Expression italienne: & $\begin{array}{l}\text { "Nella sua decisione, il giudice di seconde cure, res- } \\
\text { pinge l'appello e riconosce la competenza territoriale } \\
\text { del Tribunale di Palermo.» }\end{array}$ \\
\hline Traduction littérale: & $\begin{array}{l}\text { "Dans sa décision, le juge de second traitement } \\
\text { refuse l'appel et reconnaît la compétence territoriale } \\
\text { du Tribunal de Palerme.» }\end{array}$ \\
\hline $\begin{array}{l}\text { Traductions } \\
\text { proposées: }\end{array}$ & 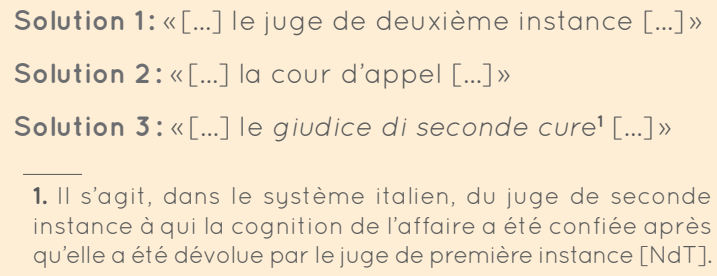 \\
\hline $\begin{array}{l}\text { Commentaires } \\
\text { des traducteurs: }\end{array}$ & $\begin{array}{l}\text { 1: «C'est ce qui se rapproche le plus dans le système } \\
\text { juridictionnel français.» } \\
\text { 3: «Ce type de juge n'existe pas dans le système } \\
\text { français.» }\end{array}$ \\
\hline
\end{tabular}

Ici, l'expression à traduire n'a pas de référent équivalent dans l'autre système juridique. Les deux solutions privilégiées par nos traducteurs sont donc l'équivalence fonctionnelle d'une part, le recours au paratexte d'autre part, avec l'utilisation de la note de bas de page.

- Des difficultés d'ordre stylistique: parmi les croyances les plus universellement répandues à l'égard du droit, figure celle selon laquelle sa phraséologie naturelle serait empreinte d'une certaine forme de noblesse. Ainsi mime-t-on souvent le personnage de l'avocat qui produit de grands effets de manches pour mieux appuyer un propos grandiloquent. Cette vision est particulièrement corroborée de l'autre côté des Alpes: Pierre Scavée parle de «complexe de Pietro Bembo'» pour décrire "l'intention esthétique [...], [le] souci d'élégance personnelle plus ou moins affirmé, mais qui s'inscrit toujours dans les normes communément

1. Pietro Bembo était un cardinal et éminent écrivain vénitien, auteur, entre autres, de Le prose della Volgar Lingua. 
admises par le goût collectif» $(1979,151)$ qui caractérisent la prose juridique italienne. Plus que d'abus de langage ou d'exercice de style, on peut évoquer la "rhétorisation» d'une langue, qu'Antoine Berman définit comme ce qui "consiste à produire des phrases "élégantes", en utilisant, pour ainsi dire, le texte de départ comme matière première» $(1999,73)$. Nos traducteurs ont souvent mentionné une prose délibérément «ennoblie» dans le texte original. Toutefois, plusieurs d'entre eux ont judicieusement fait observer que les critères et les normes stylistiques du texte juridique italien ne concordent pas avec celles du texte français: pour les émetteurs et récepteurs français, un langage soutenu - selon Antoine Berman - est caractérisé par une esthétique purifiée, une réécriture de l'original en vue d'une meilleure lisibilité, une épuration de la pesanteur et complexité de l'original en faveur du sens (lbid., p. 75). L'éloquence italienne répond à d'autres canons, correspondant plutôt à ce que Pierre Scavée définit comme «baroquisme de surcharge» (Ibid., p. 179): parmi les difficultés rencontrées, nombre de nos répondants ont en effet évoqué une pléthore de métaphores plus ou moins heureuses, une quantité excessive d'images et une intensité dans l'expression menant à une saturation qui ne saurait être acceptée sans aménagement dans la traduction française.

\begin{tabular}{|c|c|}
\hline Expression italienne: & $\begin{array}{l}\text { "Si era sviluppata una violenta guerra che aveva } \\
\text { provocato un vero bagno di sangue }[\ldots] \text { " }\end{array}$ \\
\hline Traduction littérale: & $\begin{array}{l}\text { "Une violente guerre s'était développée qui avait } \\
\text { provoqué un véritable bain de sang }[\ldots] \text { » }\end{array}$ \\
\hline \multirow[t]{2}{*}{$\begin{array}{l}\text { Traductions } \\
\text { proposées: }\end{array}$} & $\begin{array}{l}\text { Solution } 1: \ll[\ldots] \text { qui s'était soldée par de nombreux } \\
\text { morts }[\ldots] »\end{array}$ \\
\hline & Solution $2: «[\ldots]$ qui s'était terminée en massacre $[\ldots] »$ \\
\hline \multirow{2}{*}{$\begin{array}{l}\text { Commentaires } \\
\text { des traducteurs: }\end{array}$} & 1: «L'expression me semble trop forte et trop imagée.» \\
\hline & $\begin{array}{l}2 \text { : «Inapproprié au contexte, sollicite trop l'imagina- } \\
\text { tion du lectorat qui n'est pas censé être influencé.» }\end{array}$ \\
\hline
\end{tabular}


La plupart de nos répondants ont été gênés par la métaphore "bain de sang» qui renvoie à une vision trop violente qui n'a, selon eux, pas sa place dans un jugement puisqu'elle contribue à noircir le profil de l'accusé. Les traducteurs ont alors eu recours à une tactique de "débaroquisation», d'atténuation de l'effet produit, en cherchant à rendre le même concept, mais avec une autre image acoustique (Saussure, 1968, 98). S'agit-il là d'un manquement déontologique? Cette question renvoie à celle que pose Friedrich Schleiermacher de savoir s'il convient de "laisser le lecteur tranquille» et d'adapter le texte à sa culture ou au contraire de "laisser l'auteur tranquille» et de mener le lecteur à sa rencontre $(1999,48)$.

- Enfin, des difficultés d'ordre culturel: le droit et le langage qui le matérialise font partie d'un ensemble de codes non naturels imposés par la culture (Lefevere, 1992). Au même titre que leurs critères d'acceptabilité, lesdits codes diffèrent nécessairement d'une culture à l'autre. La mafia italienne étant probablement l'un des phénomènes les plus superficiellement connus et les moins bien culturellement compris des Français, biaisé par les stéréotypes qui y sont fréquemment associés, les répondants ont évoqué des difficultés à saisir pleinement le contexte culturel dans lequel s'insérait le texte de départ.

\begin{tabular}{l|l}
$\begin{array}{l}\text { Expression } \\
\text { italienne: }\end{array}$ & $\begin{array}{l}\text { "[...] È così emanato il d.l. 396/1991, il quale prevedeva } \\
\text { l'istituzione di un apposito fondo di sostegno per le } \\
\text { vittime che hanno sofferto dei danni per esserci opposti } \\
\text { alla richiesta di pizzo.» }\end{array}$ \\
\hline $\begin{array}{l}\text { Traduction } \\
\text { littérale: }\end{array}$ & $\begin{array}{l}\text { "Est ainsi émané le décret législatif } 396 / 1991, \text { qui pré- } \\
\text { voyait l'institution d'un fonds spécifiquement dédié aux } \\
\text { victimes de dommages subis après s'être opposées au } \\
\text { versement du pizzo.» }\end{array}$
\end{tabular}




\begin{tabular}{|c|c|}
\hline $\begin{array}{l}\text { Traductions } \\
\text { proposées: }\end{array}$ & $\begin{array}{l}\text { Solution 1: «[...] pour ne pas avoir cédé au racket.» } \\
\text { Solution 2: "après avoir refusé de payer l'“impôt" que } \\
\text { la mafia réclame aux commerçants.» } \\
\text { Solution } 3 \text { : «pour avoir refusé de payer le pizzo'.» } \\
\overline{\text { 1. Le pizzo est une forme de racket pratiqué par les mafias }} \\
\text { italiennes envers les commerçants locaux en échange de leur } \\
\text { «protection» [NdT]. }\end{array}$ \\
\hline $\begin{array}{l}\text { Commentaires } \\
\text { des traducteurs: }\end{array}$ & $\begin{array}{l}\text { 1: «Dans le Code pénal, le racket est défini selon les } \\
\text { mêmes termes que le pizzo italien: chantage, abus de } \\
\text { pouvoir, menace, etc.» } \\
2: \text { «Le concept de pizzo n'est pas nécessairement connu } \\
\text { en France, je l'ai donc explicité.» } \\
3: \text { "J'ai inséré une note de bas de page pour définir ce } \\
\text { qu'est le pizzo.» }\end{array}$ \\
\hline
\end{tabular}

Le premier traducteur a fait usage de ce que Zuzana Honová appelle la functional equivalence (Honová, 2016, 168), qui consiste à chercher, dans la langue et la culture cibles, un concept juridique se rapprochant au mieux de celui de la langue source. Une solution qui peut être périlleuse, car elle présente "un certain concept [qui] existe dans les deux langues, mais ne se réfère pas à la même réalité » (Idem). En l'occurrence, la notion de «racket», foncièrement négative, n'inclut pas la dimension tristement courante du pizzo qui se pratique au grand jour, est régi par des règles et sanctions instaurées par la mafia, et peut même parfois être percu comme un échange de bons procédés garantissant aux «contribuables» une prétendue protection.

Le second traducteur a quant à lui eu recours à une périphrase dont l'inconvénient est la non-conformité à la concision du texte du départ. Cette solution pose également un problème en ce qu'elle élude complètement, une fois encore, la contrepartie que propose la mafia à ses "contribuables», et donc tout un aspect culturel qui relève du genius loci.

La dernière solution, celle de la note de bas de page, alerte le lecteur sur le caractère "exotique», au sens de Lawrence Venuti $(2008,15)$, du terme problématique, et invite ainsi implicitement ce même lecteur à mener des recherches ultérieures. 


\section{Formation universitaire et propositions de traduction}

Solutions employées par les traducteurs face aux problèmes de droit comparé, selon leur formation universitaire

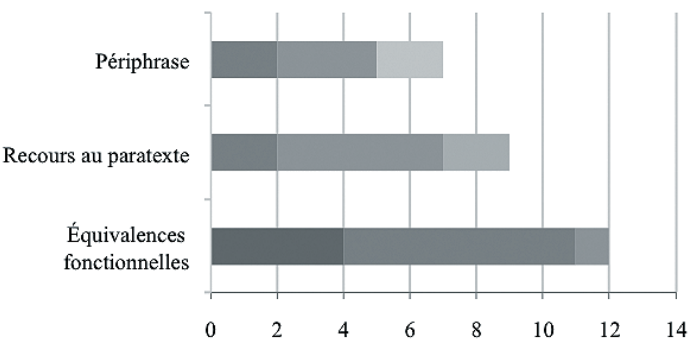

Droit

- Traduction non juridique

- Traduction juridique

Traductologie

Autres

\begin{tabular}{|l|c|c|c|}
\cline { 2 - 4 } \multicolumn{1}{c|}{} & $\begin{array}{c}\text { Équivalences } \\
\text { fonctionnelles }\end{array}$ & $\begin{array}{c}\text { Recours au } \\
\text { paratexte }\end{array}$ & Périphrase \\
\hline Droit & 4 & 0 & 0 \\
\hline$\square$ Traduction non juridique & 7 & 2 & 2 \\
\hline$\square$ Traduction juridique & 1 & 5 & 3 \\
\hline$\square$ Traductologie & 0 & 2 & 0 \\
\hline Autres & 0 & 0 & 2 \\
\hline
\end{tabular}

En cas de problèmes de droit comparé, les équivalences fonctionnelles sont très largement privilégiées, d'une part par les traducteurs ayant reçu une formation en droit, d'autre part par ceux qui ont suivi un master de traduction non juridique. Il ressort que cette pratique découle de deux procédés différents: certains de maîtriser la terminologie juridique, les juristes - qui, dans notre enquête y ont eu unanimement recours - se sentent autorisés à interpréter et à faire coïncider deux notions appartenant donc à deux systèmes notionnels distincts. L'adaptabilité au contexte cible n'est que peu prise en considération. Les répondants diplômés d'un master en traduction non juridique utilisent davantage l'équivalence fonctionnelle pour ne pas briser la linéarité de la lecture. Manquant parfois de connaissances en droit, ils peuvent également ignorer les nuances subtiles distinguant deux notions juridiques qui, bien que pouvant avoir une définition sensiblement similaire, ne s'inscrivent pas dans le même modèle de référence. Les répondants qui ont suivi une formation en 
traduction juridique semblent avoir pleine conscience de ce danger puisque seule une partie très minoritaire d'entre eux a opté pour les équivalences fonctionnelles, préférant avoir recours au paratexte et, dans une moindre mesure, à la périphrase.

Solutions employées par les traducteurs face aux problèmes d'ordre culturel, selon leur formation universitaire

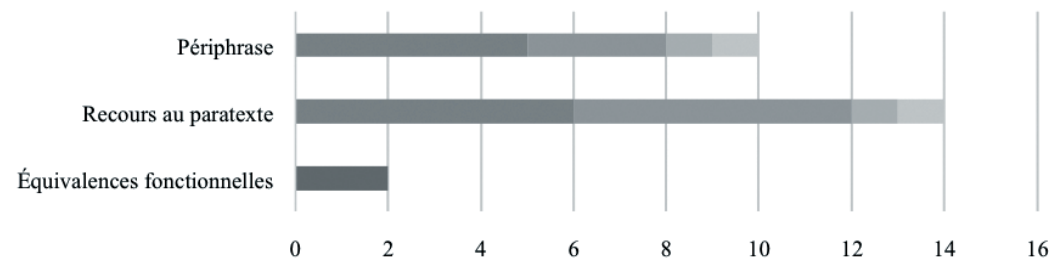

\begin{tabular}{|c|c|c|c|}
\hline & $\begin{array}{l}\text { Équivalences } \\
\text { fonctionnelles }\end{array}$ & Recours au paratexte & Périphrase \\
\hline Droit & 2 & 0 & 0 \\
\hline Traduction non juridique & 0 & 6 & 5 \\
\hline Traduction juridique & 0 & 6 & 3 \\
\hline Traductologie & 0 & 1 & 1 \\
\hline Autres & 0 & 1 & 1 \\
\hline
\end{tabular}

Droit $\quad$ Traduction non juridique $\quad$ Traduction juridique $\quad$ Traductologie $\quad$ Autres

Ici encore, on constate que la tactique des équivalences fonctionnelles a été très majoritairement privilégiée par les juristes de formation. Les répondants ayant suivi une formation en traduction, quelle qu'en soit la spécialité, ont massivement opté pour un recours au paratexte, plus particulièrement à la note de traduction, puis à la périphrase. Une nouvelle fois, on peut y voir, de la part de ces derniers, une volonté de rendre la traduction fonctionnelle et pragmatique. 


\section{Solutions employées par les traducteurs face aux problèmes} d'ordre stylistique, selon leur formation universitaire

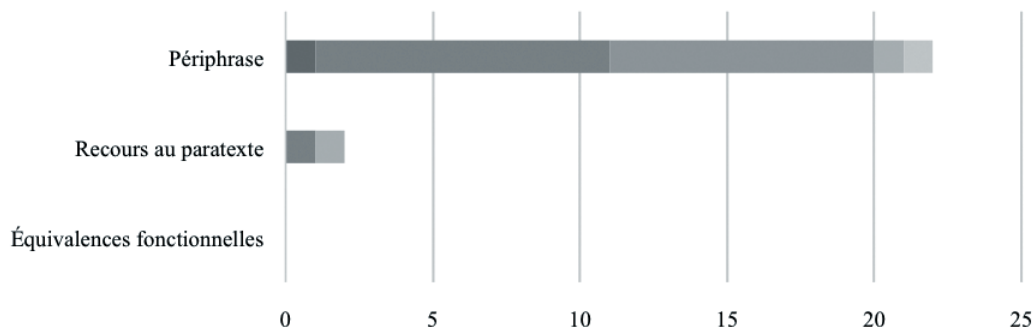

\begin{tabular}{|l|c|c|c|}
\cline { 2 - 4 } \multicolumn{1}{c|}{} & \multicolumn{1}{c|}{$\begin{array}{c}\text { Équivalences } \\
\text { fonctionnelles }\end{array}$} & Recours au paratexte & Périphrase \\
\hline Droit & 0 & 0 & 1 \\
\hline Traduction non juridique & 0 & 1 & 10 \\
\hline Traduction juridique & 0 & 0 & 9 \\
\hline Traductologie & 0 & 1 & 1 \\
\hline Autres & 0 & 0 & 1 \\
\hline
\end{tabular}

$=$ Droit $\quad$ Traduction non juridique $\quad$ Traduction juridique $=$ Traductologie $\equiv$ Autres

De ce constat se dégagent deux informations notables. D'une part, les problèmes liés à des considérations stylistiques se sont posés de façon très majoritaire aux répondants ayant reçu une formation en traduction; seuls $50 \%$ des juristes ont fait mention desdites considérations, l'autre moitié ayant privilégié une traduction littérale. D'autre part, c'est la périphrase, donc la reformulation, qui a été retenue comme la solution la plus pertinente pour $92 \%$ des répondants parmi ceux qui ont évoqué les difficultés de lexique et de phraséologie.

\section{Conclusion}

Selon Jacques Pelage (2001), "on a coutume de dire que le langage est le seul instrument du droit. Tout juriste est donc, au même titre que le traducteur, un spécialiste du langage». En revanche, il est à craindre que le «juritraducteur» ne soit pas toujours disposé à jouer un rôle de médiateur chargé d'assurer une communication entre deux cultures. Pourtant, «un texte, juridique ou non, est avant tout un instrument de 
communication», selon Michel Sparer (2002). En outre, pour David Reed, les juristes «sont par trop éloignés des problèmes linguistiques que pose le texte original» $(1979,95)$ et peuvent se retrouver en difficulté en cas de polysémies ou ambiguïtés lexicales qui, selon Gérard Cornu, représenteraient deux tiers de la terminologie juridique (2005).

L'inépuisable débat pour déterminer qui, parmi les juristes et les traducteurs, est le plus à même de traduire le droit peut trouver une proposition de réponse dans la position conciliatrice de Thomas Lebarbé pour qui «la langue du droit est par essence un domaine pluridisciplinaire de la linguistique appliquée où se croisent juristes, linguistes, informaticiens, logiciens, etc. [...]» $(2008,8)$ dont la «plus-value intellectuelle [...], se fonde sur une articulation des disciplines et un dialogue permanent entre spécialistes» (Ibid.). Stéphane Chatillon plaide lui aussi pour une collaboration entre les métiers, convaincu de l'influence réciproque qu'entretiennent langues et droits, et de leur interdépendance:

L'importance grandissante du droit international, le développement du commerce international rendent incontournable pour les juristes l'étude des droits et des langues étrangères [...]. D'un autre côté, les interprètes et les traducteurs doivent acquérir une spécialisation dans le domaine juridique, la matière étant trop technique pour qu'un "généraliste" s'y aventure sans risque pour lui et pour celvi à qui il prête son concours. $(2002,715)$

Enfin, pour Jean-Claude Gémar (1995, 9-10), le traducteur et le juriste partagent un intérêt commun matérialisé par le langage et le travail d'interprétation: si le traducteur interprète le message à restituer tandis que le juriste interprète la loi, tous deux sont mus par la même finalité qui consiste à «(re)trouver le sens» (Ibid., p. 10). La traduction est "à l'intersection du droit et de la langue.» (Ibid., p. 11) et notre enquête plaide donc pour l'acquisition d'une double compétence au moyen d'une formation universitaire en droit et en traduction.

berengere.denizeau@sorbonne-nouvelle.fr 
Bérangère Denizeau est traductrice de profession, de I'italien et de l'anglais vers le français, spécialisée dans le domaine juridique. Diplômée en 2015 du master de traduction et terminologie juridiques et financières de la Sorbonne-Nouvelle et en 2018 du master Recherche en traductologie de I'ESIT (Sorbonne-Nouvelle), elle est doctorante en juritraductologie et travaille sur l'intraduisibilité du texte législatif italien antimafia.

SOURCES CITÉES

ABDEL HADI Maher, «La juritraductologie et le problème des équivalences des notions juridiques en droit des pays arabes », in ILCEA. Revue de l'Institut des langues et cultures d'Europe, Amérique, Afrique, Asie et Australie, 3, 2002, p. 71-78.

BEAUDOIN Louis, Les Mots du droit: lexique analogique juridique, Éditions Y. Blais, 2008.

BERMAN Antoine, La Traduction et la lettre ou L'auberge du lointain, Paris, Éditions du Seuil, 1999.

CHATILLON Stéphane, «Droit et langue», in Revue internationale de droit comparé, 54, 2002, p. 687-715.

CORDONNIER Jean-Louis, Traduction et culture, Paris, Hatier-Didier, 1995.

CORNU Gérard, Linguistique juridique, Paris, Montchrestien, 2005

GÉMAR Jean-Claude, «De la traduction juridique à la jurilinguistique: la quête de l'équivalence», in Meta, 3, 2015, p. 476-493.

GÉMAR Jean-Claude, «Le plus et le moins-disant culturel du texte juridique. Langue, culture et équivalence», in Meta, 2, 2002, p. 163-176.

GÉMAR Jean-Claude, Langage du droit et traduction: essais de jurilinguistique, Montréal (Canada), Linguatech, 1982.

HONOVÁ, Zuzana, «L'équivalence fonctionnelle - une stratégie pour la traduction juridique?», in Études romanes de Brno, 2, 2016, p. 163-176.

LAVOIE Judith, «Faut-il être juriste ou traducteur pour traduire le droit? », in Meta, 3, 2003, p. 393-401.

LEBARBE Thomas, «Langue du droit, multiplicité des approches, multiplicité des disciplines », in Lidil. Revue de linguistique et de didactique des langues, 38, 2008, p. 5-9.

LEDERER Marianne, La Traduction aujourd'hui. Le modèle interprétatif, Paris, Hachette FLE, 1994.

LEFEVERE André, Translation, History, Culture: A Sourcebook, Londres, Routledge, 1992.

MONJEAN-DECAUDIN Sylvie et al., La Traduction du droit dans la procédure judiciaire: contribution à l'étude de la linguistique juridique, Paris, Dalloz, 2012. 
PELAGE Jacques, Éléments de traductologie juridique: application aux langues romanes, Fontenay-sous-Bois (France), J. Pelage, 2001.

REED David, «Problèmes de la traduction juridique au Québec », in Meta, 1, 1979, p. 95-102.

SAUSSURE Ferdinand de, Cours de linguistique générale. Tome 1, Wiesbaden (Allemagne), Otto Harrassowitz, 1968.

SCAVÉE Pierre et INTRAVAIA Pietro, Traité de stylistique comparée : analyse comparative de litalien et du français, Bruxelles, 1979.

SCHLEIERMACHER Friedrich Daniel Ernst, Des différentes méthodes du traduire, Paris, Éditions du Seuil, 1999.

SPARER Michel, «Peut-on faire de la traduction juridique? Comment doit-on l'enseigner?», in Meta, 2, 2002, p. 266-278.

TERRAL Florence, «L'empreinte culturelle des termes juridiques», in Meta, 49, 2004, p. 876-890.

VENUTI Lawrence, The Translator's Invisibility: A History of Translation, Londres, Routledge, 2008. 